\title{
The Synaptophysin-Synaptobrevin Complex: a Hallmark of Synaptic Vesicle Maturation
}

\author{
Anja Becher, ${ }^{1}$ Anne Drenckhahn, ${ }^{1}$ Ingrid Pahner, ${ }^{1}$ Martin Margittai, ${ }^{2}$ Reinhard Jahn, ${ }^{2}$ and \\ Gudrun Ahnert-Hilger ${ }^{1}$ \\ 1/nstitut für Anatomie der Charité, Humboldt-Universität zu Berlin, 10115 Berlin, Germany, and ${ }^{2 M a x}$ Planck Institut für \\ Biophysikalische Chemie, 37077 Göttingen, Germany
}

\begin{abstract}
Exocytosis of synaptic vesicles requires the formation of a fusion complex consisting of the synaptic vesicle protein synaptobrevin (vesicle-associated membrane protein, or VAMP) and the plasma membrane proteins syntaxin and soluble synaptosomal-associated protein of $25 \mathrm{kDa}$ (or SNAP 25). In search of mechanisms that regulate the assembly of the fusion complex, it was found that synaptobrevin also binds to the vesicle protein synaptophysin and that synaptophysin-bound synaptobrevin cannot enter the fusion complex. Using a combination of immunoprecipitation, cross-linking, and in vitro interaction experiments, we report here that the synaptophysinsynaptobrevin complex is upregulated during neuronal development. In embryonic rat brain, the complex is not detectable, although synaptophysin and synaptobrevin are expressed and are localized to the same nerve terminals and to the same pool of vesicles. In contrast, the ability of synaptobrevin to participate in the fusion complex is detectable as early as embryonic day 14 . The binding of synaptoporin, a closely
\end{abstract}

Exocytosis of synaptic vesicles is mediated by a conserved set of membrane proteins that are commonly referred to as SNAREs [soluble $\mathrm{N}$-ethylmaleimide-sensitive factor attachment protein (SNAP) receptors]. These proteins include synaptobrevin [or vesicle-associated membrane protein (VAMP)], which is localized in the membrane of synaptic vesicles, and syntaxin 1 and SNAP 25, which are predominantly localized at the synaptic plasma membrane. In vitro, these proteins spontaneously form a tight stoichiometric complex that can be disassembled by interaction with the proteins $\alpha$-SNAP and the ATPase $N$-ethylmaleimide-sensitive factor (NSF) (Söllner et al., 1993). The cyclic assembly-disassemby of SNARE proteins is one of the crucial steps in exocytotic membrane fusion. The core of the ternary complex consists of a rod-shaped long bundle of helices, with all membrane anchors aligned at one end (Hanson et al., 1997a; Sutton et al., 1999). Based on these studies, a picture is emerging according to which SNARE assembly results in the tight binding of the vesicle to the plasma membrane, largely overcoming the energy barrier for fusion (Hanson et al., 1997b; Jahn and Hanson, 1998).

\footnotetext{
Received Sept. 10, 1998; revised Dec. 11, 1998; accepted Dec. 22, 1998.

This work was supported by Deutsche Forschungsgemeinschaft Grant SFB 515. We thank Evelyn Heuckendorf for expert technical assistance.

Correspondence should be addressed to Dr. Gudrun Ahnert-Hilger, Institut für Anatomie der Charité, Humboldt-Universität zu Berlin, Philippstraße 12, 10115 Berlin, Germany.

Copyright (ㄷ) 1999 Society for Neuroscience $\quad 0270-6474 / 99 / 191922-10 \$ 05.00 / 0$
}

related homolog of synaptophysin, to synaptobrevin changes in a similar manner during development. Recombinant synaptobrevin binds to synaptophysin derived from adult brain extracts but not to that derived from embryonic brain extracts. Furthermore, the soluble cytosol fraction of adult, but not of embryonic, synaptosomes contains a protein that induces synaptophysin-synaptobrevin complex formation in embryonic vesicle fractions. We conclude that complex formation is regulated during development and is mediated by a posttranslational modification of synaptophysin. Furthermore, we propose that the synaptophysin-synaptobrevin complex is not essential for exocytosis but rather provides a reserve pool of synaptobrevin for exocytosis that can be readily recruited during periods of high synaptic activity.

Key words: synaptophysin-synaptobrevin complex; SNARE proteins; synaptic vesicles; fine-tuning of exocytosis; synapse maturation; neuronal development

Much less is known about how the formation of the ternary complex is regulated. Because assembly occurs spontaneously and is irreversible in the absence of NSF and $\alpha$-SNAP, a tight control of this assembly reaction may be instrumental for intracellular membrane traffic. Therefore, special attention has been given recently to proteins that selectively bind to one of the SNAREs and thus prevent it from interacting with its SNARE partners. For syntaxin, the best candidate for such a control protein is the protein munc-18, the mammalian version of Caenorhabditis elegans unc-18, which is known to be essential for exocytosis. Syntaxin that is complexed to munc-18 appears to be unable to interact with SNAP 25 and synaptobrevin (Hata et al., 1993; Okamoto and Südhof, 1997). Furthermore, numerous other proteins were identified that selectively interact with syntaxin 1 , such as munc-13-1 (Betz et al., 1997), but in most cases the functional significance of these interactions remains to be established. For synaptobrevin, only one group of non-SNARE binding partner proteins is known: the membrane protein synaptophysin and its closely related isoform synaptoporin. Synaptophysins are major residents of the synaptic vesicle membrane. The binding to synaptobrevin is specific and exclusive; synaptobrevin bound to synaptophysin cannot enter the SNARE complex and, conversely, synaptobrevin, which is part of a SNARE complex, cannot interact with synaptophysin (Calakos and Scheller, 1994; Edelmann et al., 1995). These findings led to the proposal that synaptophysin serves as a control protein that regulates the availability of synaptobrevin for engaging with its SNARE partners before fusion. 
However, direct evidence for such a regulatory role is lacking. Furthermore, the nature and the potential regulation of the synaptophysin-synaptobrevin interaction are still unknown.

In the present study, we have attempted to learn more about the significance of the synaptophysin-synaptobrevin interaction for neuronal exocytosis. Specifically, we have investigated whether this interaction is a basic feature of all synaptobrevindependent membrane fusion events or whether it exists solely in fully differentiated presynaptic terminals. During neuronal development, most components of the exocytotic machinery are expressed long before differentiated synapses are established (Fletcher et al., 1991; Matteoli et al., 1995; Ahnert-Hilger et al., 1996). Our findings show that during these early phases, synaptophysin is unable to bind to synaptobrevin but acquires binding capability during later developmental stages parallel to synaptogenesis. Furthermore, we found that this change is caused by a still unknown modification of synaptophysin that is mediated by a synaptosomal cytosolic factor present in adult brain.

This article contains part of the PhD thesis of Anja Becher.

\section{MATERIALS AND METHODS}

Antibodies. The following antibodies were described previously: mouse monoclonal antibodies against synaptobrevin II (clone 69.1; Edelmann et al., 1995); synaptophysin (clone 7.2; Jahn et al., 1985); synaptotagmin (clone 41.1; Brose et al., 1992); and rabbit polyclonal antisera against synaptophysin (Jahn et al., 1985). The hybridoma line HPC-1 (syntaxin 1) was kindly provided by C. Barnstable (Yale University, Hartford, CT) (Barnstable et al., 1985) and later grown from our own stocks. Rabbit sera directed against the following proteins were kind gifts: synaptobrevin (provided by T. Rapoport, Harvard Medical School, Boston, MA) (Kutay et al., 1995); synaptoporin (provided by T. C. Südhof, Howard Hughes Medical Institute, University of Texas, Dallas, TX); and $\alpha$-subunit of the potassium channel Kv1.6 (provided by R. Veh, Institut für Anatomie der Charité, Humboldt-Universität Berlin, Germany) (Veh et al., 1995). A monoclonal antibody against SNAP 25 was purchased from Sternberger Monoclonals (Baltimore, MD). Secondary antibodies for Western blot detection, horse anti-mouse, and goat antirabbit, conjugated with either horseradish peroxidase or alkaline phosphatase, were purchased from Vector Laboratories (Burlingame, CA). For immunocytochemical detection, goat anti-mouse labeled with Cy5 (Jackson ImmunoResearch, West Grove, PA) and goat anti-rabbit labeled with Cy2 (Amersham, Arlington Heights, IL) were used.

Synaptosomes. Isolated nerve terminals (crude synaptosomes) were prepared at $4^{\circ} \mathrm{C}$ from adult and embryonic rat whole brains in the presence of protease inhibitors (Edelmann et al., 1995). After homogenization in $0.32 \mathrm{M}$ sucrose (nine strokes at $900 \mathrm{rpm}$ ) and centrifugation at $2,500 \times g$ for $2 \mathrm{~min}$, the supernatants were centrifuged at $13,000 \times g$ for $12 \mathrm{~min}$. Crude synaptosomes (1-2 mg of protein) were dissolved in 1 $\mathrm{ml}$ extraction buffer containing (in mM): KCl 140, EDTA 2, HEPES$\mathrm{KOH} \mathrm{20,} \mathrm{pH} 7.3$, and 1\% (v/v) Triton X-100. Extraction was performed for $1 \mathrm{hr}$ at $4^{\circ} \mathrm{C}$, followed by centrifugation for $3 \mathrm{~min}$ at $700 \times g$. Three microliters of ascites fluid (corresponding to $\sim 10 \mu \mathrm{g}$ of $\mathrm{IgG}$ ) of the monoclonal antibodies against synaptobrevin, synaptophysin, or syntaxin were added to $200 \mu \mathrm{l}$ of extraction supernatant. Incubation was performed for 16-18 hr. Immunoprecipitates were separated by addition of $25 \mu \mathrm{l}$ of G-Sepharose suspension (Pharmacia Biotech, Piscataway, NJ). The beads were collected by centrifugation at $200 \times g$ for $1 \mathrm{~min}$, washed three times in extraction buffer, and analyzed by SDS-PAGE and Western blotting. The supernatant of the immunoprecipitate was analyzed in parallel.

For cross-linking experiments, synaptosomes were resuspended in Krebs'-Ringer's buffer containing (in mM): $\mathrm{NaCl} 140, \mathrm{NaHCO}_{3} 5$, $\mathrm{MgCl}_{2} 1, \mathrm{Na}_{2} \mathrm{HPO}_{4}$ 1.2, glucose 10, and HEPES-NaOH 20, pH 7.4. Synaptosomes $[1.5 \mathrm{mg} / \mathrm{ml}$ protein for adult, $4 \mathrm{mg} / \mathrm{ml}$ for embryonic day 20 (E20)] were prewarmed at room temperature for $10 \mathrm{~min}$. The chemical cross-linker disuccinimidyl suberate (DSS) dissolved in dimethyl sulfoxide was added to yield a final concentration of $0.5 \mathrm{~mm}$. After incubation at room temperature for $45 \mathrm{~min}$, while shaking, the reaction was quenched by the addition of Tris- $\mathrm{HCl}, \mathrm{pH} 7.4$ (final concentration
$100 \mathrm{~mm}$ ) and incubated for an additional $30 \mathrm{~min}$. Membranes were subsequently pelleted at $350,000 \times g$ for $30 \mathrm{~min}$ and analyzed by SDSPAGE and immunoblotting using nondenaturating conditions.

Synaptic vesicles and synaptosomal cytosol. Crude synaptic vesicles [lysis pellet 2 (LP2) fraction] were prepared from adult and embryonic brains following the procedure described by Huttner et al. (1983). The resulting supernatant [lysis supernatant 2 (LS2)] was used as synaptosomal cytosol. For protein digestion, $0.2 \mathrm{mg} / \mathrm{ml}$ trypsin was added during LS2 sample preparation and inactivated before incubation with LP2.

Immunoisolation. Brains were homogenized in $0.32 \mathrm{M}$ sucrose $(2000$ rpm, 10 strokes) and spun down at 35,000 $\times g$ for $25 \mathrm{~min}$. Three hundred microliters of the resulting supernatant were incubated with $1-3 \mu \mathrm{l}$ of a suspension of Eupergit C1Z beads (Roehm Pharma, Weiterstadt, Germany) coupled to monoclonal antibodies against synaptobrevin, synaptophysin, or glycine (as control) for $45 \mathrm{~min}$ at $4^{\circ} \mathrm{C}$. Beads were centrifuged at $7,500 \times g$ for $2 \mathrm{~min}$, and the pellets were washed three times (Walch-Solimena et al., 1995). Proteins in the bead pellet and the supernatant were analyzed by SDS-PAGE and Western blotting.

Cell culture. PC 12 cells were cultivated in DMEM supplemented with $10 \%$ horse and $5 \%$ fetal calf serum. Before use, cells were harvested, washed in PBS, and pelleted.

Recombinant synaptobrevins. Full-length rat synaptobrevin II (residues 1-116) was subcloned into the NdeI and EcoRI sites of the vector $\mathrm{pHO} 2 \mathrm{c}$ (Fasshauer et al., 1997). Synaptobrevin II without the transmembrane region (residues 1-96) was subcloned into TrcHisA (Invitrogen, San Diego, CA) as described previously (Chapman et al., 1994). The His6tagged fusion proteins were purified by $\mathrm{Ni}^{2+}$-Sepharose chromatography and eluted by a stepwise increase of the imidazole concentration. The eluate was analyzed by SDS-PAGE and Coomassie blue staining. Recombinant synaptobrevins were immobilized on Ni-beads via their His tag and used for binding studies with synaptophysin from adult and embryonic vesicles and from PC 12 cells. Binding was performed in PBS containing 3-[(3-cholamiolopropyl)dimethylammonio-1-propane sulfonic acid $(1.5 \% \mathrm{w} / \mathrm{v})$ or Triton X-100 $(1 \% \mathrm{v} / \mathrm{v})$. Incubations were performed for $16-18 \mathrm{hr}$, after which the beads were pelleted and washed three times. Proteins in the bead pellet, as well as the supernatant, were analyzed by SDS-PAGE and Western blotting.

Immunocytochemistry. Freshly removed cerebella from E21 or adult rats were placed in ice-cold PBS and cut into small cubes of $\sim 8 \mathrm{~mm}^{3}$. These were fixed in $4 \%$ formaline in $0.1 \mathrm{~m}$ phosphate buffer, $\mathrm{pH} 7.4$, for $1 \mathrm{hr}$ on ice and $2 \mathrm{hr}$ at room temperature and subsequently immersed in $30 \%$ sucrose in PBS overnight. The tissue was shock-frozen in tissuefreezing medium (Jung Leica, Nussloch, Germany). Ten micrometer sections were prepared using a cryostat and mounted on poly-D-lysine (Sigma, St. Louis, MO)-coated slides. After three rinses with PBS, sections were incubated with a blocking solution containing $5 \%$ normal goat serum (Pan Systems, Nuerenberg, Germany) and 2\% bovine serum albumin, pH 7.0 (fraction V; Serva Feinbiochemica, Heidelberg, Germany) dissolved in PBS supplemented with $0.1 \%$ Triton X-100 for $1 \mathrm{hr}$ at room temperature. Incubation with a mixture of polyclonal and monoclonal antibodies against synaptobrevin and synaptophysin diluted in blocking solution was performed overnight at $4^{\circ} \mathrm{C}$. Immunofluorescence was detected with Cy5-labeled goat anti-mouse and Cy2-labeled goat anti-rabbit and analyzed by confocal laser microscopy.

\section{RESULTS}

\section{Synaptophysin-synaptobrevin complex and SNARE complex in adult and embryonic brains}

Synaptobrevin occurs in two mutually exclusive complexes: the SNARE complex and a complex with synaptophysin. Using coimmunoprecipitation, we first analyzed these two complexes in synaptosomes from embryonic brain, starting with E14, compared with synaptosomes from adult brain. In adult synaptosomes, the monoclonal antibody against synaptobrevin coimmunoprecipitated the two other SNARE proteins, syntaxin and SNAP 25, as well as synaptophysin. The antibody against syntaxin precipitated only synaptobrevin and SNAP 25, leaving synaptophysin in the supernatant, and the synaptophysin antibody clearly precipitated 
adult

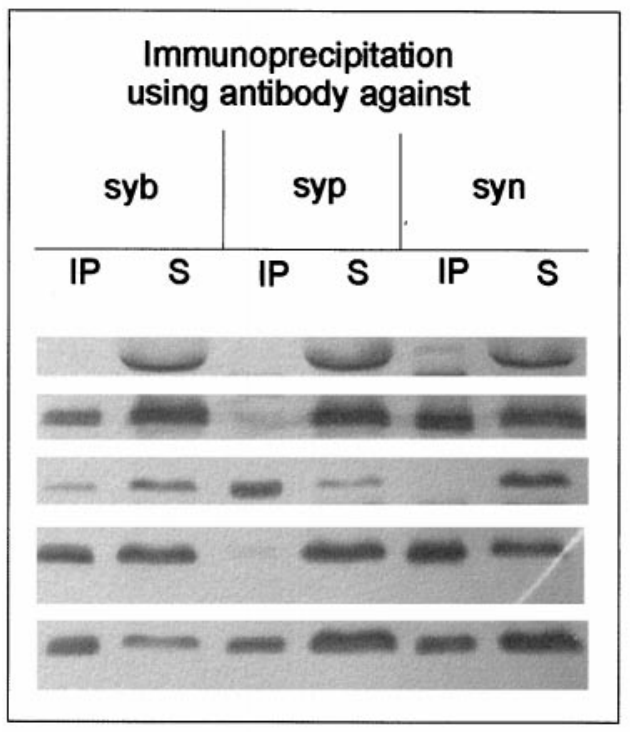

ED 14

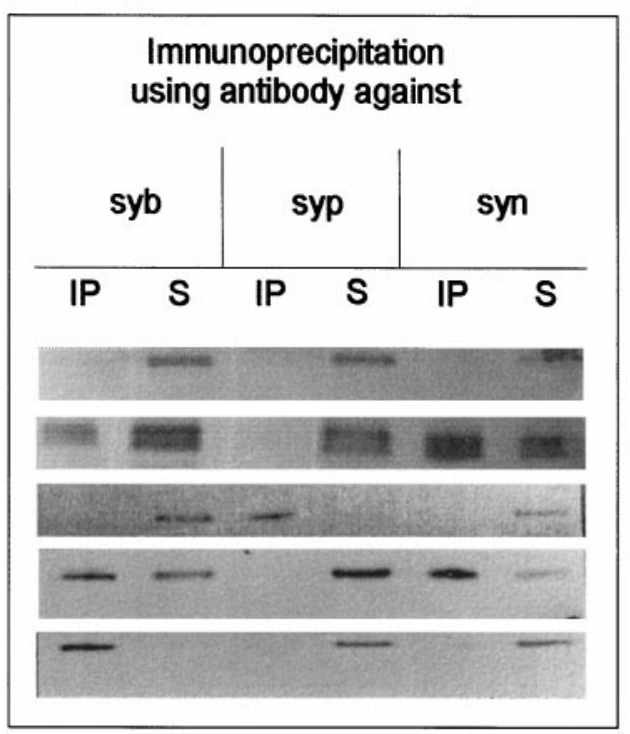

adult, SDS-treated

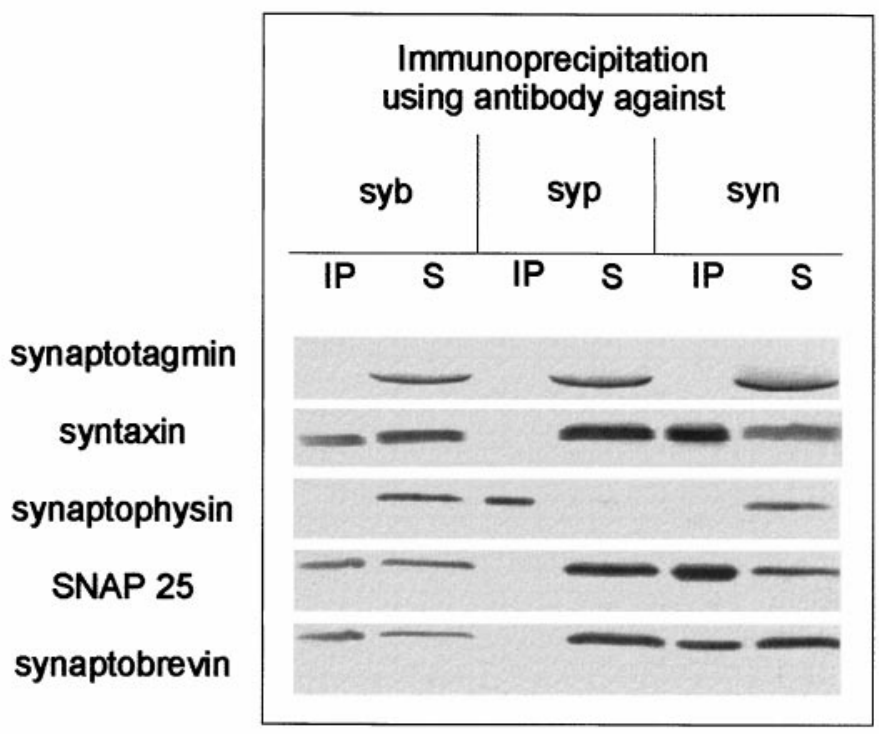

ED 20

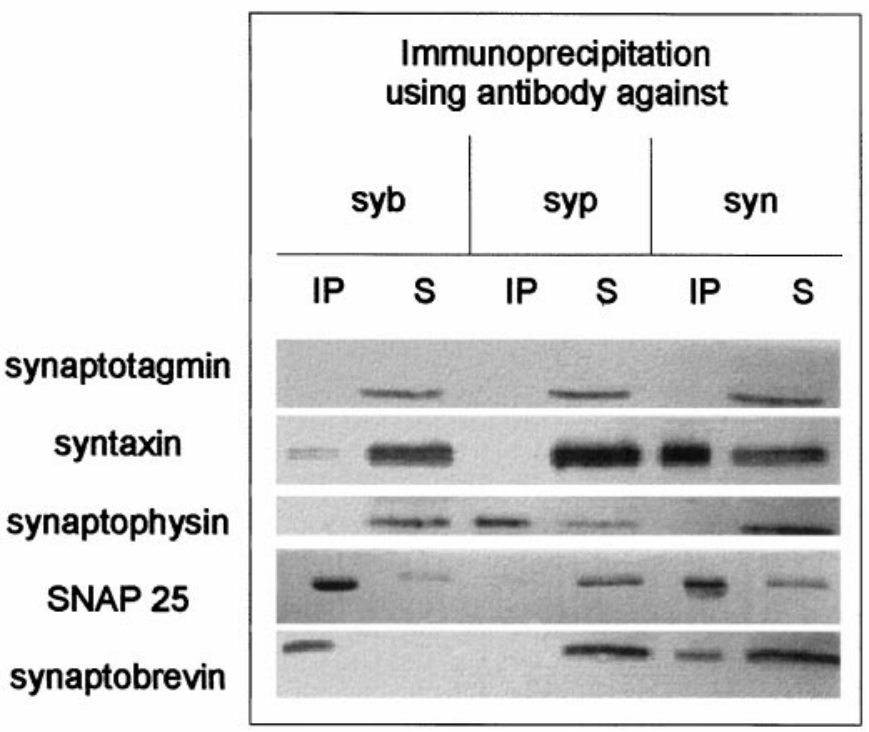

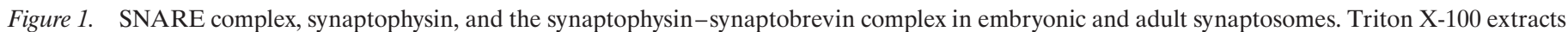

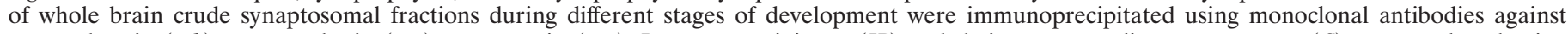

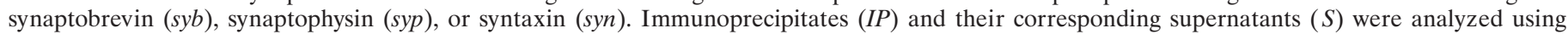
antibodies against the indicated proteins.

only synaptobrevin. The synaptophysin-synaptobrevin complex dissociated when synaptosomes were treated with SDS. These findings are in agreement with previous reports (Calakos and Scheller, 1994; Edelmann et al., 1995). However, when synaptosomes from E14 or E20 were used, the synaptophysin-synaptobrevin complex could not be detected when immunoprecipitated with antibodies against synaptophysin or synaptobrevin, although synaptophysin could clearly be seen in the supernatants after precipitation with the synaptobrevin or the syntaxin antibody, respectively. In contrast, the antibodies specific for syntaxin and synaptobrevin precipitated assembled SNARE complexes (Fig. $1)$, demonstrating that synaptobrevin is not impaired in its ability to enter such complexes. In both cases, synaptotagmin, an integral membrane protein colocalizing with synaptobrevin to synaptic vesicles, was always found in the supernatant (Fig. 1, top two panels).

Varying the ratio between protein amount and Triton X-100 gave similar results, excluding that detergent artifacts caused the lack of synaptophysin-synaptobrevin interaction in embryonic samples (data not shown). The synaptophysin-synaptobrevin complex could not be detected in embryonic synaptosomes, even when the amount of protein for immunodetection was increased approximately tenfold (data not shown). The difference in the synaptophysin-synaptobrevin complex between adult and embry- 


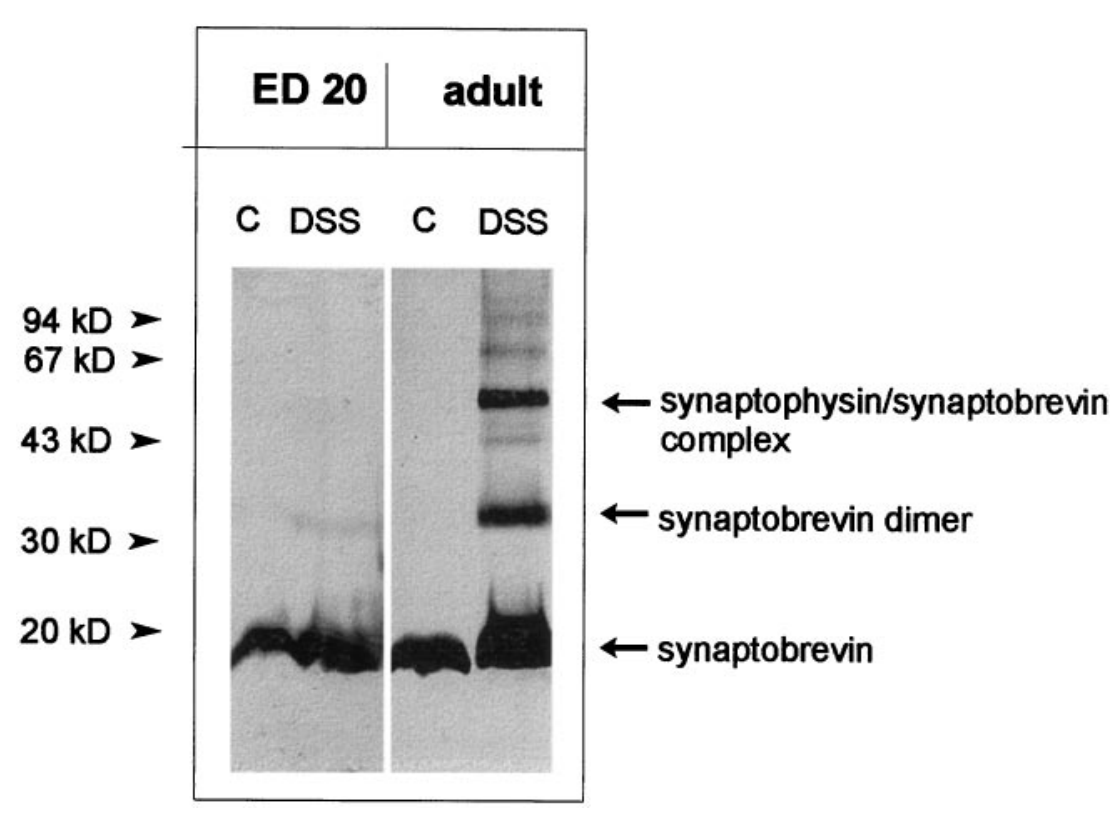

Figure 2. Complexes of synaptophysin and synaptobrevin after chemical cross-linking in embryonic and adult synaptosomes. Crude synaptosomal fractions from embryonic day $(E D) 20$ whole brain $(4 \mathrm{mg} / \mathrm{ml}$ protein) or adult whole brain $(1.5 \mathrm{mg} / \mathrm{ml}$ protein) were treated with disuccinimidyl suberate $(D S S)$ as described in Materials and Methods. After SDS-PAGE and Western blotting, membranes were analyzed using the monoclonal antibody against synaptobrevin. Note that cross-linking reveals the synaptobrevin dimer and the synaptobrevinsynaptophysin complex in the adult synaptosomes, whereas no synaptobrevin-synaptophysin complex and only traces of the synaptobrevin dimer can be detected in embryonic synaptosomes. $C$, Control.

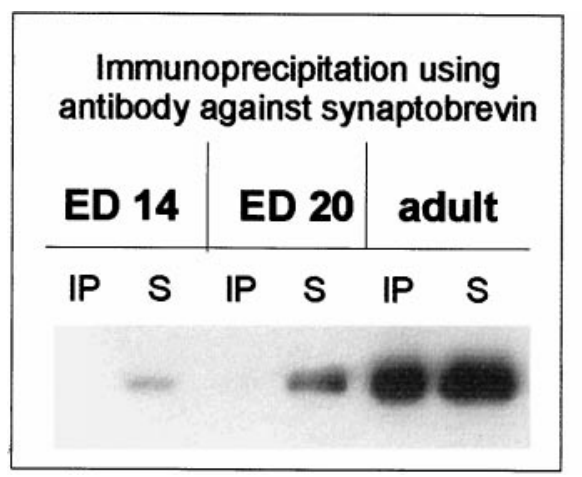

synaptoporin

Figure 3. Synaptoporin and the synaptoporin-synaptobrevin complex in embryonic and adult synaptosomes. Triton X-100 extracts of whole brain crude synaptosomal fractions during different stages of development were immunoprecipitated using the monoclonal antibody against synaptobrevin. Immunoprecipitates $(I P)$ and their corresponding supernatants $(S)$ were analyzed using an antiserum against the synaptophysin analog synaptoporin.

onic brains was also observed when using polyclonal antisera for immunodetection (data not shown).

To confirm the absence of synaptophysin-synaptobrevin complexes in embryonic brains by an independent approach, we incubated synaptosomes with the cross-linker DSS. This crosslinker is membrane-permeable and allows for an analysis of the complex in intact membranes, i.e., without the need for detergent solubilization (Johnston and Südhof, 1990; Calakos and Scheller, 1994; Edelmann et al., 1995). When synaptosomes from adult brain were used, DSS treatment resulted in the generation of two adducts of $\sim 36$ and $56 \mathrm{kDa}$, which represent the synaptobrevin dimer and the synaptobrevin-synaptophysin complex, respectively. In embryonic synaptosomes, no adduct corresponding to the synaptophysin-synaptobrevin complex was present. Furthermore, only small amounts of the synaptobrevin dimer were detected (Fig. 2). A similar pattern was obtained when immunoprecipitation with both synaptobrevin- and synaptophysin antibodies was performed before electrophoretic analysis (data not shown).

Next, we investigated whether the second isoform of synapto-

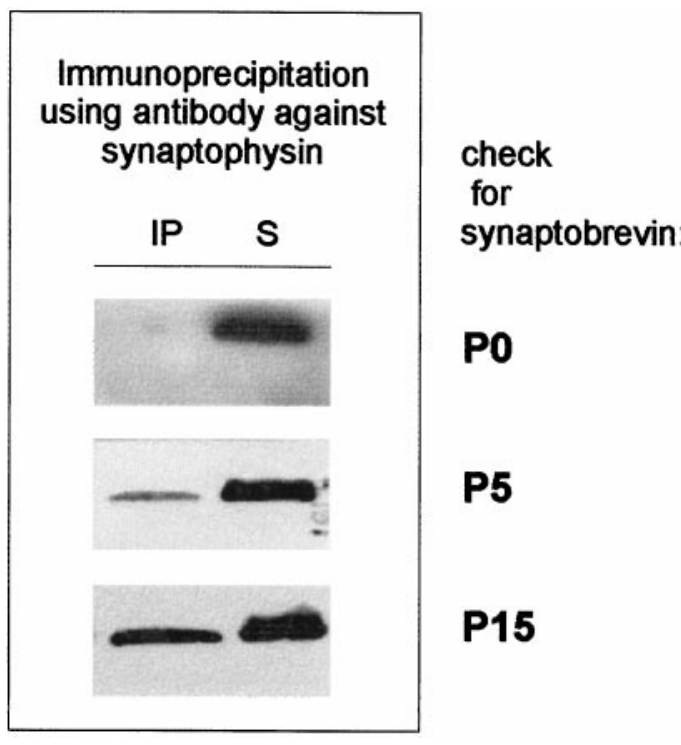

Figure 4. Postnatal appearance of the synaptophysin-synaptobrevin complex. Triton X-100 extracts of whole brain crude synaptosomal fractions from different stages of postnatal development were immunoprecipitated using the monoclonal antibody against synaptophysin. Immunoprecipitates $(I P)$ and their corresponding supernatants $(S)$ were analyzed using an antibody against synaptobrevin.

physin, synaptoporin, shows a comparable pattern of complex formation with synaptobrevin. In embryonic synaptosomes, synaptoporin could also be detected as early as E14. Like synaptophysin, synaptoporin did not form complexes with synaptobrevin in embryonic synaptosomes, whereas, as shown previously (Edelmann et al., 1995), the synaptoporin-synaptobrevin complex was present in adult synaptosomes (Fig. 3).

Next, we tested for the presence of the synaptophysin-synaptobrevin complex at several early postnatal time points, starting at birth. As shown in Figure 4, the synaptophysin-synaptobrevin complex appeared only after birth, starting at approximately postnatal day $2-5$ and reaching adult levels at approximately postnatal day 15 . 


\section{A cerebellum ED 18}

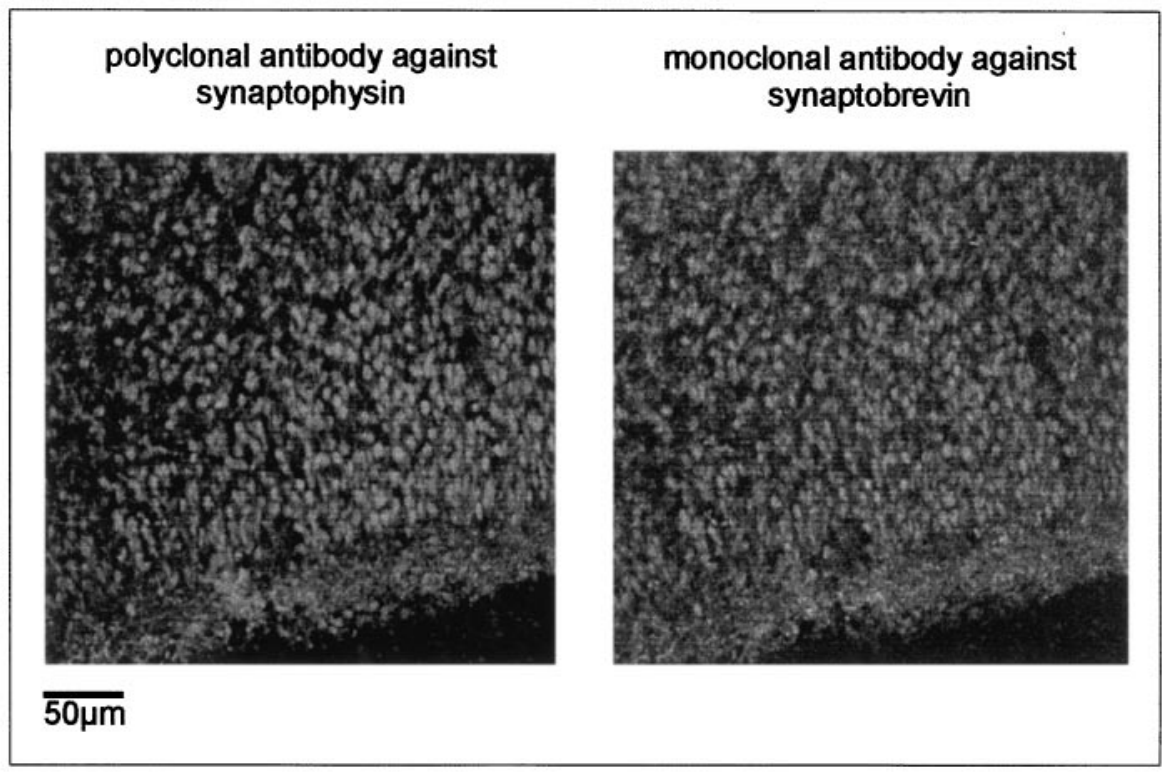

Figure 5. Synaptophysin, synaptobrevin, and the synaptophysin-synaptobrevin complex in embryonic and adult cerebellum. A, Confocal laser microscopic analysis of synaptophysin and synaptobrevin in embryonic day 18 rat cerebellar cortex. Note the clear presence of synaptophysin (left) and synaptobrevin (right) in the developing molecular and granular layers and the almost complete colocalization of both antigens. $B$, Triton X-100 extracts of cerebellar crude synaptosomal fraction from embryonic day 18 or adult were immunoprecipitated using antibodies against synaptophysin or synaptobrevin. Immunoprecipitates $(I P)$ and their corresponding supernatants $(S)$ were analyzed using anti-synaptophysin or anti-synaptobrevin antibody. No synaptobrevin-synaptophysin complex could be detected in the embryonic cerebellar synaptosomes.

\section{B cerebellum ED 20}

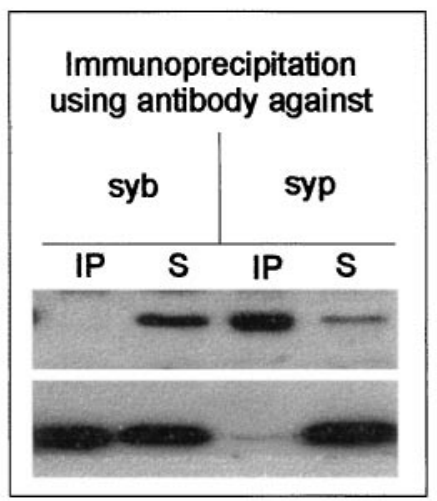

\section{cerebellum adult}

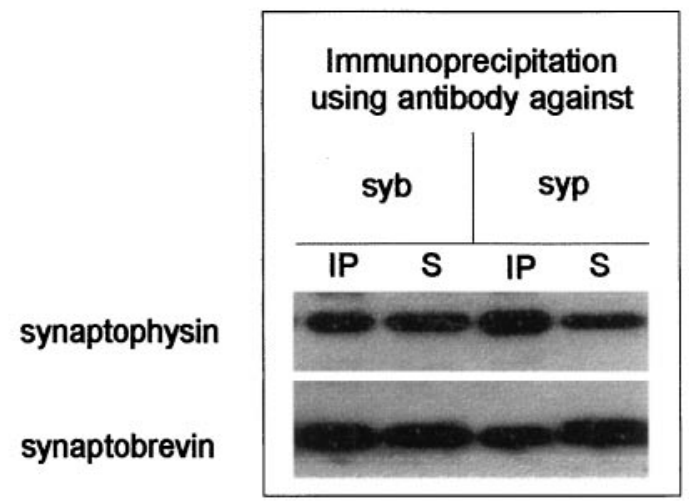

\section{Failure of synaptophysin to complex synaptobrevin in embryonic neurons is not attributable to a differential sorting}

The data discussed so far shows that in embryonic brain synaptophysin and synaptobrevin are expressed but fail to complex. An obvious explanation for this observation may be that the two proteins do not reside on the same vesicle population during early development. For instance, the proteins may first be expressed preferably in different subsets of neurons. Alternatively, the proteins may reside in the same neurons but may localize to different membrane compartments before the formation of mature synapses. To test for regional differences in protein expression, we analyzed the distribution of synaptophysin and synaptobrevin in slices from adult and embryonic cerebella using double-label immunocytochemistry (Fig. 5A). In the cerebellar cortex of E18 rats, both proteins showed a complete colocalization in the molecular layer and the granular layer. This resembles the colocal- ization of both proteins in adult cortex (Baumert et al., 1989) and excludes differential expression as the cause for the absence of the complex. Immunoprecipitation from adult and embryonic cerebella reflected the results obtained in whole brain (Fig. $5 B$ ).

Although the immunocytochemical data demonstrate that both synaptophysin and synaptobrevin are coexpressed in the same neurons as early as E18, it cannot be excluded that the two proteins are localized to different and complementary vesicle subpopulations that cannot be differentiated at the light microscopic level. To test for this possibility, we immunoisolated synaptophysin-containing vesicles from adult and embryonic brains using Eupergit beads coated with synaptophysin antibodies. This procedure was shown previously to result in the one-step isolation of membranes, with exceptional purity (Burger et al., 1989; Walch-Solimena et al., 1995). As shown in Figure 6, vesicles isolated by this procedure contained both synaptophysin and synaptobrevin, regardless of whether they were obtained from 
ED 20

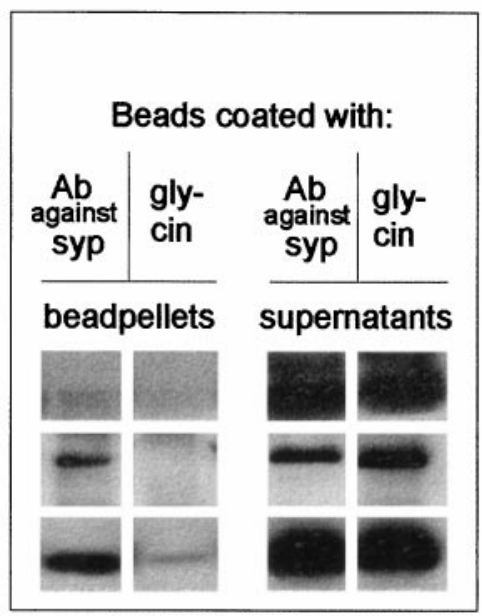

Syb 1-116

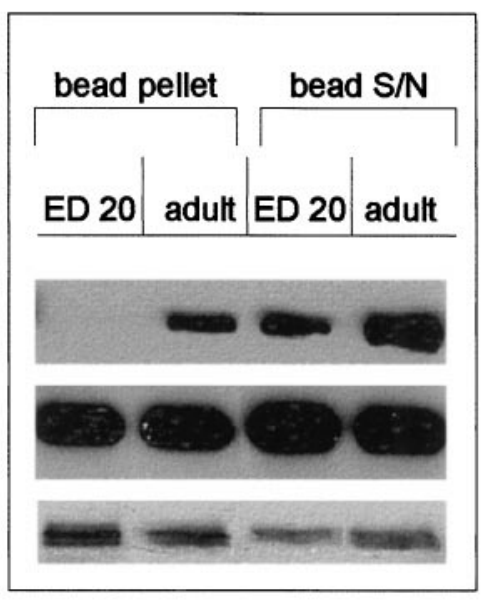

adult

\section{Beads coated with:}

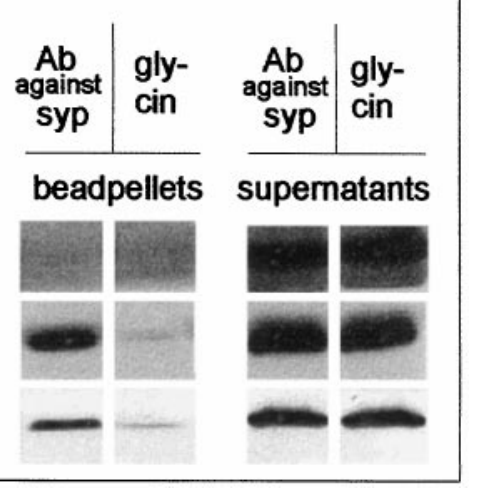

Figure 6. Immunoisolation of synaptic vesicles from embryonic and adult whole brain. Eupergit beads coated with either an antibody against synaptophysin or glycine as negative control were incubated with crude synaptic vesicle fractions as described in Materials and Methods. Membranes adsorbed to the beads, as well as the corresponding supernatants, were analyzed using antibodies against synaptobrevin, synaptophysin, or the $\alpha$ subunit of the Kv1.6 channel. Only beads coated with the antibody against synaptophysin pelleted synaptobrevin and synaptophysin from both embryonic and adult vesicles. The potassium channel protein was always detected in the supernatant. synaptophysin

synaptobrevin

\section{Syb 1-96}

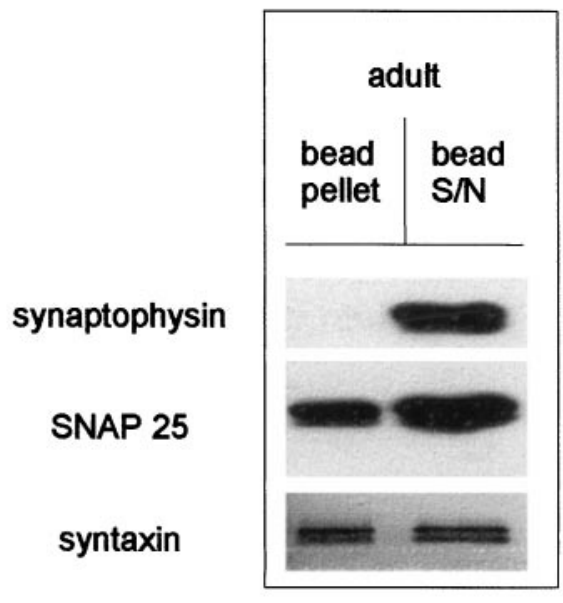

Figure 7. Binding of recombinant synaptobrevin constructs to embryonic and adult synaptic vesicles. Crude synaptic vesicle extracts from embryonic or adult brain were incubated with either full-length synaptobrevin (1$116)$ or the N-terminal part of synaptobrevin (1-96) (see Materials and Methods). The bead pellets and the corresponding supernatants were analyzed using antibodies against synaptophysin, SNAP 25, and syntaxin. adult or embryonic brain. Neither synaptophysin nor synaptobrevin was detectable on control beads devoid of antibody. As an independent control, we also tested for the presence of the $\alpha$ subunit of the potassium channel Kv1.6, an integral membrane protein that is known to be localized to the plasma membrane. This protein did not bind to the beads and was found only in the supernatants of the immunoprecipitates (Fig. 6). These data demonstrate that synaptophysin and synaptobrevin occur on the same vesicle in both adult and embryonic brain and that their failure to complex is not because of a differential localization in embryonic neurons.

\section{Association is regulated by a change in synaptophysin during development that is controlled by a cytosolic protein}

The data described above indicates that the synaptophysin-synaptobrevin interaction is directly regulated on the surface of the synaptic vesicle and their precursor membranes. Such regulation may be achieved by either a direct posttranslational modification of one or both of the proteins, or an interaction between an unknown control protein and one of the binding partners. To pinpoint the mechanism for the developmental change, we first investigated which of the two proteins differs in its ability to interact with its partner. Recombinant synaptobrevin was gener- ated by bacterial expression and incubated with detergent extracts derived from both embryonic and adult brain. As shown in Figure 7, only synaptophysin from adult synaptosomes bound to synaptobrevin, whereas synaptophysin from embryonic synaptosomes did not. In contrast, SNAP 25 and syntaxin from both preparations bound to recombinant synaptobrevin (Fig. 7A). As shown previously (Edelmann et al., 1995), no binding was observed when only the cytoplasmic portion of synaptobrevin was used, whereas the binding of the SNARE partners was not affected (Fig. 7B). These data strongly suggest that the biochemical properties of synaptobrevin remain unchanged during development and resemble that of bacterially expressed synaptobrevin. We conclude that embryonic synaptophysin differs from adult synaptophysin.

In the neuroendocrine cell line PC 12, the synaptophysinsynaptobrevin complex was also absent, although both proteins were present, as shown by immunoprecipitation with the relevant antibodies (Fig. 8A). Again, as in embryonic neurons, PC 12 synaptophysin was unable to bind recombinant synaptobrevin, although the SNARE proteins SNAP 25 and syntaxin were clearly able to interact (Fig. $8 B$ ).

We next investigated whether synaptosomal cytosol prepared from adult brain induces formation of the synaptophysin-synap- 


\section{A PC12}

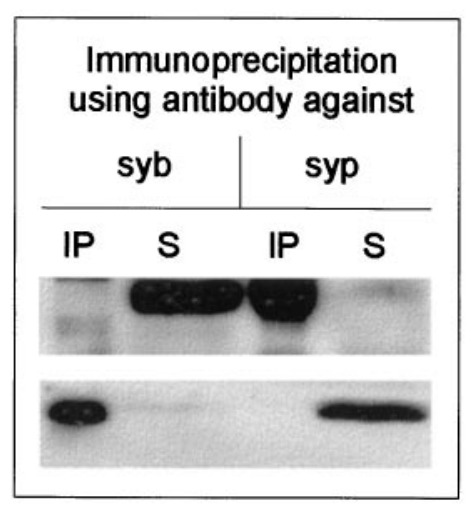

B

synaptophysin

synaptobrevin
Syb 1-116

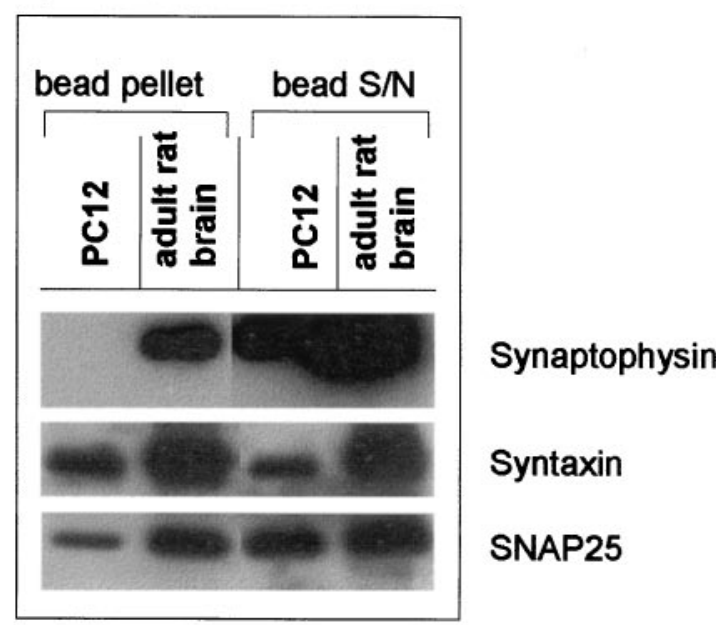

Figure 8. Absence of synaptophysin-synaptobrevin complex in PC 12 cells. A, Triton X-100 extracts of PC 12 cells were immunoprecipitated using monoclonal antibodies against synaptobrevin $(s y b)$ and synaptophysin $(s y p)$. Immunoprecipitates $(I P)$ and their corresponding supernatants $(S)$ were analyzed using antibodies against synaptophysin and synaptobrevin. B, PC 12 cell extracts and crude synaptic vesicle extracts were incubated with full-length synaptobrevin $(1-116)$. The bead pellets and the corresponding supernatants were analyzed using antibodies against synaptophysin, SNAP 25 , and syntaxin.

tobrevin complex in synaptic vesicles from embryonic brain. Cytosolic fractions from embryonic and adult synaptosomes (LS2) were prepared by ultracentrifugation. When synaptic vesicles prepared from embryonic brain were incubated with adult synaptosomal cytosol, binding between synaptophysin and synaptobrevin was induced. No binding was observed when the vesicles were incubated with cytosol prepared from embryonic brain synaptosomes (Fig. 9A, left lanes). Incubation with an embryonic synaptosomal cytosolic preparation did not affect the synaptophysin-synaptobrevin complex of adult vesicles (Fig. 9A, right lanes), nor was binding observed when the embryonic vesicles were incubated with buffer instead of with embryonic synaptosomal cytosol (Fig. 9B). Note that the synaptosomal cytosol was completely free of both synaptophysin and synaptobrevin (Fig. $9 B$ ). Trypsin-digested adult synaptosomal cytosol was no longer able to induce synaptophysin-synaptobrevin complex formation on embryonic vesicles (Fig. 9C), strongly suggesting that the active factor of the adult synaptosomal cytosol is a protein. We conclude that the ability of synaptophysin to bind to synaptobrevin is regulated by a posttranslational modification that is mediated by a cytosolic protein. This protein is absent or inactive in embryonic brain but is upregulated during synaptogenesis.

\section{DISCUSSION}

In the present study, we have shown that synaptophysin-synaptobrevin complexes are upregulated during development. Before synaptogenesis, the proteins do not interact, despite the fact that they reside on the same vesicle population. Using recombinant synaptobrevin as an exogenous binding partner, we have shown further that the lack of interaction is caused by an unknown modification of synaptophysin. Finally, we provide evidence that the interaction can be induced by a cytosolic protein that is expressed or active only in adult brain.

Several lines of evidence document that in immature neurons synaptobrevin is fully functional with respect to exocytotic membrane fusion. First, the ability of synaptobrevin to form SNARE complexes does not change during development. Fully assembled
SNARE complexes can be precipitated from immature neurons as soon as the SNARE proteins are expressed. Second, it has been demonstrated previously that hypothalamic and cerebellar neurons grown for $3 \mathrm{~d}$ in culture are able to secrete GABA by activity-dependent and tetanus toxin-sensitive exocytosis (Ahnert-Hilger et al., 1996; Kraszewski et al., 1996). Thus, synaptobrevin-dependent exocytosis of synaptic vesicles or their precursors can be observed long before mature synapses form. Third, in the neuroendocrine cell line PC 12, which undergoes regulated and synaptobrevin-dependent exocytosis, synaptophy$\sin$ is unable to complex with synaptobrevin. It should be kept in mind, however, that elongation of neurites appears to be independent of synaptobrevin (Sweeney et al., 1995; Ahnert-Hilger et al., 1996), although it is mediated by exocytotic incorporation of precursor vesicles (Fletcher et al., 1991, 1994). In contrast, deletion of SNAP 25 (Osen-Sand et al., 1993) or syntaxin (Igarashi et al., 1996) by neurotoxin cleavage impairs axonal differentiation. Thus, it is possible that the exocytotic events underlying membrane growth during differentiation and those underlying regulated release of neurotransmitters are separately regulated.

If the ability of synaptophysin to complex with synaptobrevin is not an essential prerequisite for synaptobrevin-dependent exocytosis, what then could be the role of this interaction? Although other explanations cannot be excluded at present, we believe that synaptophysin may fine-tune synaptic responses by regulating the availability of synaptobrevin to the SNARE complex in a positive manner. According to this view, the main role of complex formation is to provide a rapidly available pool of vesicular synaptobrevin for exocytotic membrane fusion. Synaptophysin prevents the association of synaptobrevin with vesicle-associated syntaxin and SNAP 25, which are known to reside in significant quantities on synaptic vesicles (Walch-Solimena et al., 1995) and to form complexes with synaptobrevin (Otto et al., 1997). Such vesicular SNARE complexes probably represent a "dead-end" status because synaptobrevin would require another round of ATP- and NSF-driven disassembly to reacquire functionality in exocytosis. 
A

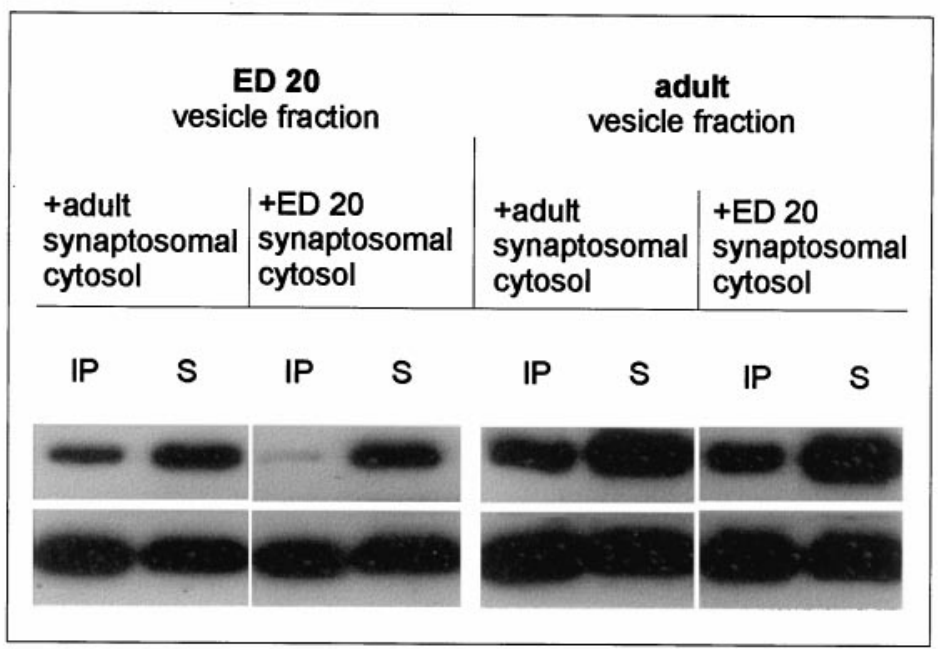

B

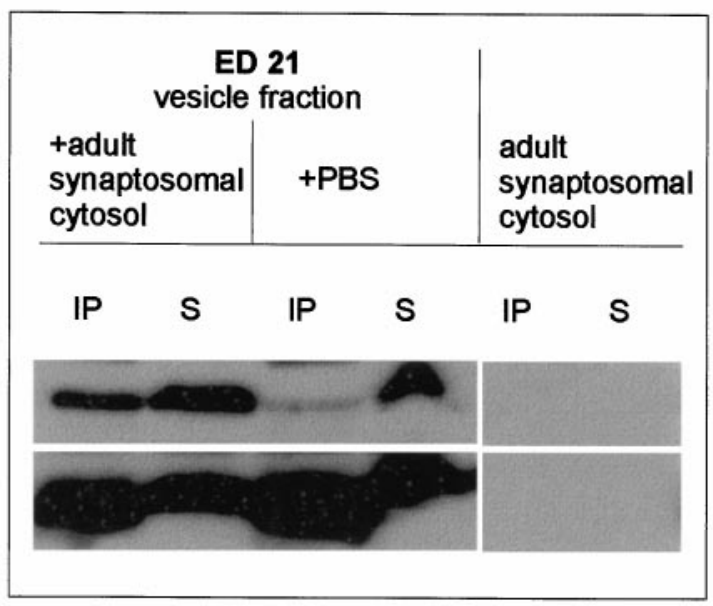

synaptophysin

synaptobrevin

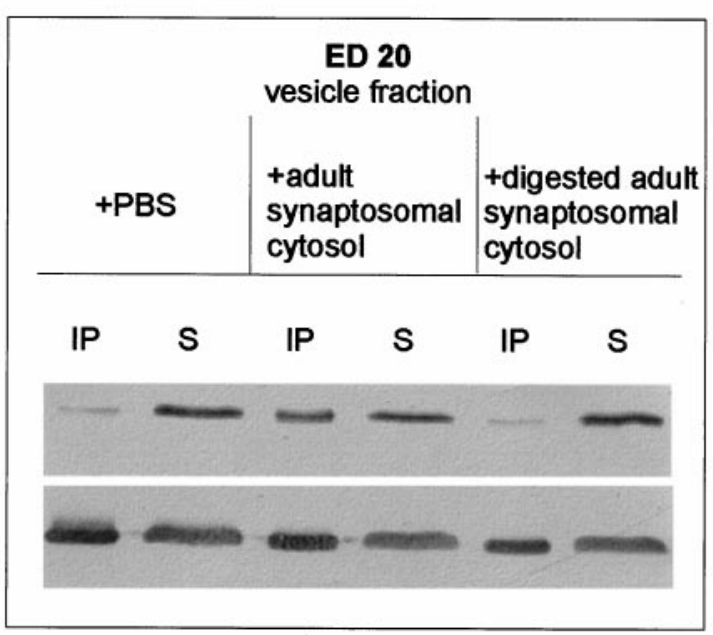

synaptophysin

synaptobrevin synaptophysin

synaptobrevin
Figure 9. Induction of the synaptophysin-synaptobrevin complex in embryonic synaptic vesicles by adult cytosol. $A$, Crude synaptic vesicle fractions from embryonic day 20 or adult brain were incubated with synaptosomal cytosol fractions (LS2, synaptosomal cytosol) obtained from either adult (left lanes) or embryonic (ED 20, right lanes) brain for $90 \mathrm{~min}$ at $37^{\circ} \mathrm{C}$ before they were subjected to extraction and immunoprecipitation procedures using the antisynaptobrevin antibody. Immunoprecipitates $(I P)$ and their corresponding supernatants $(S)$ were analyzed using anti-synaptophysin or anti-synaptobrevin antibody. $B$, Crude synaptic vesicle fractions from embryonic day 21 brain were incubated with either PBS or LS2 (synaptosomal cytosol) obtained from adult brains and processed as described in $A$. Note that the embryonic cytosol is completely free of both synaptophysin and synaptobrevin (right lanes). C, Crude synaptic vesicle fractions from embryonic day 20 were incubated with either PBS or with undigested or trypsin-digested LS2 (synaptosomal cytosol) obtained from adult brains. Samples were extracted, immunoprecipitated, and analyzed as described in $A$. 
In fact, the relative instability of the synaptophysin-synaptobrevin complex may ensure that its formation is readily reversible when synaptobrevin is needed to enter the SNARE complex during regulated exocytosis. In this way, synaptophysin would recruit synaptobrevin after disassembly of the SNARE complex after vesicle endocytosis by keeping the extremely reactive synaptobrevin (Fasshauer et al., 1997) from entering the SNARE complex in an uncontrolled manner and releasing it when it is required for another round of rapid exocytosis. Support for such a role is also provided by the observation that overexpression of synaptophysin enhances neurotransmitter release (Alder et al., 1995).

The molecular mechanism underlying the synaptophysin-synaptobrevin interaction remains to be established. The presence of both proteins on the same vesicles in embryonic brain excludes a differential sorting during development, a process that has been suggested previously for PC 12 cells (Bauerfeind and Huttner, 1993). Homodimerization of synaptobrevin (Laage and Langosch, 1997), which is observed primarily in adult brain (Fig. 2), appears not to play a role in the synaptophysin-synaptobrevin interaction seeing that cross-linking data seem to suggest a complex of synaptophysin with one rather than two synaptobrevins. Further experiments are necessary, however, to determine the exact stoichiometry of the synaptophysin-synaptobrevin complex. Our data strongly suggest that the binding is regulated by a direct posttranslational modification of synaptophysin, but its nature is still unknown. According to our preliminary data, protein phosphorylation of synaptophysin appears not to be involved. Similarly, regulation is apparently not mediated by a rearrangement of disulfide bonds in synaptophysin. When the disulfide bonds are reduced by SH-agents, the synaptophysin-synaptobrevin complex falls apart but readily reassembles once the sulfhydril reagents are removed. However, such treatment fails to induce synaptobrevin-binding in embryonic synaptophysin (our unpublished observations). The cytosolic protein capable of inducing formation of the synaptophysin-synaptobrevin complex is currently under investigation.

Mice that do not express synaptophysin nevertheless exhibit functional neurotransmission, indicating that synaptophysin is not an absolute requirement for neurotransmitter release as such (Eshkind and Leube, 1995; McMahon et al., 1996), although synaptophysin isoforms, such as synaptoporin, may substitute at least partially for synaptophysin in these mice. So far, the data on members of the synaptophysin family (Janz and Südhof, 1998) suggest that synaptophysin and its analogs may be involved in fine-tuning of neurotransmitter release in adult neurons and probably in sorting and vesicle biogenesis in developing neurons. Our findings reported here provide a clue as to how such fine control may be achieved. The developmental profile of the synaptophysin-synaptobrevin interaction, leading from a less controlled to a highly regulated synaptobrevin, may enable synaptic vesicles to adjust to the high efficiency of exocytosis in differentiated synapses.

\section{REFERENCES}

Ahnert-Hilger G, Kutay U, Chahoud I, Rapoport TA, Wiedenmann B (1996) Synaptobrevin is essential for secretion but not for the development of synaptic processes. Eur J Cell Biol 70:1-11.

Alder J, Kanki H, Valtorta F, Greengard P, Poo MM (1995) Overexpression of synaptophysin enhances neurotransmitter secretion at $\mathrm{Xe}$ nopus neuromuscular synapses. J Neurosci 15:511-519.
Barnstable CJ, Hofstein R, Akagawa K (1985) A marker of early amacrine cell development in rat retina. Brain Res 352:286-290.

Bauerfeind R, Huttner WB (1993) Biogenesis of constitutive secretory vesicles, secretory granules and synaptic vesicles. Curr Opin Cell Biol [Erratum 5:1106] 5:628-635.

Baumert M, Maycox PR, Navone F, DeCamilli P, Jahn R (1989) Synaptobrevin: an integral membrane protein of 18,000 daltons in small synaptic vesicles. EMBO J 8:379-384.

Betz A, Okamoto M, Benseler F, Brose N (1997) Direct interaction of the rat unc-13 homologue munc-13-1 with the $\mathrm{N}$ terminus of syntaxin. J Biol Chem 272:2520-2526.

Brose N, Petrenko AG, Südhof TC, Jahn R (1992) Synaptotagmin: a calcium sensor on synaptic vesicle surface. Science 256:1021-1025.

Burger PM, Mehl E, Maycox PR, Baumert M, Lottspeich F, DeCamilli P, Jahn R (1989) Synaptic vesicles immunoisolated from rat cerebral cortex contain high levels of glutamate. Neuron 3:715-720.

Calakos N, Scheller RH (1994) Vesicle-associated membrane protein and synaptophysin are associated on the synaptic vesicle. J Biol Chem 269:24534-24537.

Chapman ER, An S, Barton N, Jahn R (1994) SNAP-25, a t-SNARE which binds both syntaxin and synaptobrevin via domains that may form coiled coils. J Biol Chem 269:27427-27432.

Edelmann L, Hanson PI, Chapman ER, Jahn R (1995) Synaptobrevin binding to synaptophysin: a potential mechanism for controlling exocytotic fusion machine. EMBO J 14:224-231.

Eshkind LG, Leube RE (1995) Mice lacking synaptophysin reproduce and form typical synaptic vesicles. Cell Tissue Res 282:423-433.

Fasshauer D, Otto H, Eliason WK, Jahn R, Brünger AT (1997) Structural changes are associated with the soluble $N$-ethylmaleimidesensitive fusion protein attachment protein receptor complex formation. J Biol Chem 272:28036-28041.

Fletcher TL, Cameron P, DeCamilli P, Banker G (1991) The distribution of synapsin I and synaptophysin in hippocampal neurons developing in culture. J Neurosci 11:1617-1626.

Fletcher TL, DeCamilli P, Banker G (1994) Synaptogenesis in hippocampal cultures: evidence indicating that axons and dendrites become competent to form synapses at different stages of neuronal development. J Neurosci 14:6695-6706.

Hanson PI, Roth R, Morosaki H, Jahn R, Heuser JE (1997a) Structure and conformational changes in NSF and ist membrane receptor complexes visualized by quick-freeze/deep-etch electron microscopy. Cell 90:523-535.

Hanson PI, Heuser,JE, Jahn R (1997b) Neurotransmitter release-four years of SNARE complexes. Curr Opin Neurobiol 7:310-315.

Hata Y, Slaughter GH, Südhof TC (1993) Synaptic vesicle fusion complex contains unc-18 homologue bound to syntaxin. Nature 366:347-351.

Huttner WB, Schiebler W, Greengard P, DeCamilli P (1983) Synapsin I (protein I), a nerve terminal-specific phosphoprotein. III. Its association with synaptic vesicles studied in a highly purified synaptic vesicle preparation. J Cell Biol 96:1374-1388.

Igarashi M, Kozaki S, Terakawa S, Kawano S, Ide C, Komiya Y (1996) Growth-cone collapse and inhibition of neurite growth induced by botulinum neurotoxin $\mathrm{C} 1$ : a t-SNARE is involved in axonal growth. J Cell Biol 134:205-215.

Jahn R, Hanson PI (1998) Membrane fusion. SNAREs line up in new environment. Nature 393:14-15.

Janz R, Südhof TC (1998) Cellugyrin, a novel ubiquitous form of synaptogyrin that is phosphorylated by pp60c-src. J Biol Chem 273:2851-2857.

Jahn R, Schiebler W, Oimet C, Greengard P (1985) A 38,000 dalton membrane protein (p38) present in synaptic vesicles. Proc Natl Acad Sci USA 82:4137-4141.

Johnston PA, Südhof TC (1990) The multisubunit structure of synaptophysin. Relationship between disulfide bonding and homooligomerization. J Biol Chem 265:8869-8873.

Kraszewski K, Daniell L, Mundigl O, DeCamilli P (1996) Mobility of synaptic vesicles in nerve endings monitored by recovery from photobleaching of synaptic vesicle-associated fluorescence. J Neurosci 16:5905-5913.

Kutay U, Ahnert-Hilger G, Hartmann E, Wiedenmann B, Rapoport TA (1995) Transport route for synaptobrevin via a novel pathway of insertion into the endoplasmic reticulum membrane. EMBO J 14:217-223. 
Laage R, Langosch D (1997) Dimerization of the synaptic vesicle protein synaptobrevin (vesicle-associated membrane protein) II depends on specific residues within the transmembrane domain. Eur J Biochem 249:540-546.

Matteoli M, Viderio C, Kraszewski K, Mundigl O, Coco S, Fumagalli G, DeCamilli P (1995) Mechanisms of synaptogenesis in hippocampal neurons in primary culture. J Physiol (Paris) 89:51-55.

McMahon HT, Boshakov VY, Janz R, Hammer RE, Siegelbaum SA, Südhof TC (1996) Synaptophysin, a major vesicle protein, is not essential for neurotransmitter release. Proc Natl Acad Sci USA 93:4760-4764.

Okamoto M, Südhof TC (1997) Munc-18 interacting proteins in synaptic vesicle exocytosis. J Biol Chem 272:31459-31464.

Osen-Sand A, Catsicas M, Staple JK, Jones KA, Ayala G, Knowles J, Grenningloh G, Catsicas S (1993) Inhibition of axonal growth by SNAP-25 antisense oligonucleotides in vitro and in vivo. Nature 364:445-448.

Otto H, Hanson PI, Jahn R (1997) Assembly and disassembly of ternary complex of synaptobrevin, syntaxin and SNAP-25 in the membrane of synaptic vesicles. Proc Natl Acad Sci USA 94:6197-6201.

Söllner T, Whiteheart SW, Brunner M, Erdjument-Bromage H, Geromanos S, Tempst T, Rothman JE (1993) SNAP receptors implicated in vesicle targeting and fusion. Nature 362:318-324.

Sutton B, Fasshauer D, Jahn R, Brünger AT (1998) Crystal structure of a core synaptic fusion complex at 2.4 A resolution. Nature 395:347-353.

Sweeney ST, Broadie K, Keane J, Niemann H, O'Kane CJ (1995) Targeted expression of tetanus toxin light chain in Drosophila specifically eliminates synaptic transmission and causes behavioural defects. Neuron 14:341-351.

Veh RW, Lichtinghagen R, Sewing S, Wunder F, Grumbach IM, Pongs O (1995) Immunocytochemical localization of five members of the Kv1 channel subunits: contrasting subcellular locations and neuron-specific co-localizations in rat brain. Eur J Biochem 7:2189-2205.

Walch-Solimena C, Blasi J, Edelmann L, Chapman ER, Fischer von Mollard G, Jahn R (1995) The t-SNAREs syntaxin and SNAP-25 are present on organelles that participate in synaptic vesicle recycling. J Cell Biol 128:637-645. 